\title{
Maturation and Patency Rates in Basilic Transposition Arteriovenous Fistula Under Regional Versus General Anesthesia: A Single- Center, Retrospective, Observational Study
}

\author{
Waryam Muhammad Saleh ${ }^{1,2}$, Zia U. Rehman ${ }^{1}$, Shiraz Hashmi ${ }^{3}$ \\ 1. Section of Vascular Surgery, Department of Surgery, Aga Khan University Hospital, Karachi, PAK 2. Department of \\ Vascular and Endovascular Surgery, Shaheed Mohtarma Benazir Bhutto Institute of Trauma, Karachi, PAK 3. \\ Department of Surgery, Aga Khan University Hospital, Karachi, PAK
}

Corresponding author: Waryam Muhammad Saleh, panhwar.waryam145@gmail.com

\section{Abstract \\ Background}

Basilic transposition arteriovenous fistula (BT AVF) is a viable option for dialysis-dependent patients, which can be performed under either general or regional anesthesia. Regional anesthesia is reported to cause vascular dilatation during the perioperative period, leading to improved fistula success. Regional anesthesia is also considered safe as compared to general anesthesia in terms of hemodynamic stability. Limited and conflicting data are available comparing regional versus general anesthesia in terms of fistula maturation and patency. We aimed to compare the maturation, one-year patency rates, and complication rates in patients undergoing single-stage BT AVF in regional versus general anesthesia.

\section{Methods}

This retrospective observational study was conducted on patients undergoing single-stage BT AVF from January 2016 to December 2019. Patients were divided into regional (RA) vs. general anesthesia (GA) groups and compared in terms of maturation, one-year patency, and perioperative complication rates.

\section{Results}

Out of 152 patients, 110 (72.37\%) were in GA while 42 (27.63\%) were in the RA group. Elderly, female, diabetic, ischemic heart disease, and American Society of Anesthesiologists (ASA) class IV patients were more in the RA group. Other comorbid and vascular access-related factors were comparable between the groups. A trend toward higher maturation rates (97.6\% vs. $92.1 \%$ ) and one-year patency rates (62.5\% vs. $56.6 \%$ ) was observed in the RA vs. GA group, however, the difference did not attain statistical significance, $\mathrm{p}=0.359$ and $\mathrm{p}=0.327$, respectively. The rate of access abandonment was higher in the GA group ( $43.4 \%$ vs. $37.5 \%)$. The most prevalent cause of abandonment was death in the RA group while it was access failure in

Review began 07/14/2021 Review ended 07/26/2021 Published 08/08/2021

๑) Copyright 2021

Muhammad Saleh et al. This is an open access article distributed under the terms of the Creative Commons Attribution License CC-BY 4.0., which permits unrestricted use, distribution, and reproduction in any medium, provided the original author and source are credited. the GA group. Overall complication rates were comparable between both groups ( $20.2 \%$ vs. $17.5 \%$, p=0.816).

\section{Conclusion}

Regional anesthesia is a useful technique with potentially improved maturation and patency rates. Nevertheless, an assumed benefit of regional anesthesia in terms of anesthesia-related complications was not observed.

Categories: Anesthesiology, Cardiac/Thoracic/Vascular Surgery, Nephrology

Keywords: basilic transposition arteriovenous fistula, regional anesthesia, maturation, patency, complication rates

\section{Introduction}

Chronic kidney disease (CKD) is a debilitating disease and significant public health problem, affecting $12 \%$ $18.7 \%$ of the population in Europe as well as Asia [1-2]. The prevalence of CKD is as high as $23.3 \%$ in Pakistan [3]. Patients with end-stage renal disease (ESRD) need renal replacement therapy in form of renal transplant or dialysis. A significant proportion of these patients cannot undergo a renal transplant and need lifelong dialysis. Good quality, durable angioaccess is a major contributing factor in determining survival in this sub-set of the CKD population. An arteriovenous fistula (AVF) is considered the best vascular access because of longer patency rates and lower intervention and complication rates [4-7].

Radiocephalic AVF (RC AVF) and brachiocephalic AVF (BC AVF) are considered primary and preferred autologous accesses. A basilic transposition arteriovenous fistula (BT AVF), first described in 1976 by Dagher et al., has been increasingly accepted as a viable option for secondary vascular access [8] and it is indicated when adequate superficial veins in the forearm or arm are not available for primary access procedures. It has higher venous flow with high patency and maturation rates but BT AVF needs longer operative time and 
longer dissection of basilic vein for its superficialization and transposition, requiring either general (GA) or regional anesthesia (RA). BT AVF is performed either as single-stage if the vein is good-sized, but if it is of smaller size, initially only brachio-basilic AVF is formed in the first stage and superficialization of AVF is done at the second stage, four to six weeks after the initial procedure [9].

In general anesthesia (GA) patients are made unconscious with or without muscle relaxation with the placement of an endotracheal tube or laryngeal mask airway (LMA) while in RA local anesthetic agent is infiltrated around nerves, thus making the area supplied by these nerves numb. For BT AVF RA is administered through a supraclavicular or infraclavicular brachial plexus blockage, usually under ultrasound guidance. ESRD patients are known to be at increased risk of perioperative and postoperative complications [10]. GA is considered riskier than RA mainly because of stress induction that eventually causes hypotension leading to decreased blood flow and thrombosis of fistula [11]. On the contrary, RA is a good alternative, as it avoids hemodynamic instability and stress response compared to GA and causes venous and arterial dilatation by its sympathectomy effect [12]. This vasodilatory effect of RA results in increased venous diameter and peripheral flow intraoperatively and several hours after surgery, thus avoiding perioperative vascular spasm and possibly decreased early access failure and improved maturation and patency rates [13-14]. However, no consensus exists over the optimal mode of anesthesia for AVF creation and it is not clear whether this vasodilatory effect translates into favorable outcomes in terms of improved maturation and patency rates. Although evidence suggests improved patency and maturation and decreased early failure rates when RA is compared with LA [15], data are scarce on the comparative outcomes of RA versus GA, especially on BT AVF formation.

We hypothesize that due to the vasodilatory effect, RA may result in improved outcomes of AVF compared to GA patients undergoing BT AVF. A retrospective, observational study was designed to compare maturation and patency rates at one year as the primary outcome and perioperative complications as the secondary outcome in patients undergoing single-stage BVT AVF under RA or GA.

\section{Materials And Methods}

This is a retrospective study conducted on adult patients (aged 18 years or above), undergoing single-stage BT AVF under GA or RA, at the Section of Vascular Surgery, Department of Surgery, Aga Khan University Hospital, Karachi, Pakistan, from January 2016 to December 2018. Patients were recruited by the consecutive, non-probability sampling technique. The cohort was divided into the GA and RA groups. Both groups were followed postoperatively till one year from index surgery for maturation, patency rates at one year, and complication rates. Patients who underwent two-stage brachiobasilic AVF, with missing data or loss of follow-up, underwent BT AVF surgery under local anesthesia with or without sedation were excluded from the study analysis.

Data were collected after getting approval from the institutional ethical review committee (ERC\# 2020-560114962). The data were extracted from charts and clinical notes on a prestructured proforma.

Information on demographics, including age, gender, and body mass index (BMI), comorbid conditions, and vascular risk factors, such as diabetes, hypertension, hyperlipidemia, ischemic heart disease, tobacco use, and American society of anesthetists (ASA) class, were recorded from clinic notes, anesthesia preoperative assessment forms, and admission notes. Factors affecting fistula maturation and patency such as cause of renal failure, dialysis dependency, previous history of access surgery, previous central cannulation, and size of the basilic vein and brachial artery were also recorded. Data on type of anesthesia (RA or GA), the success of RA, and any anesthesia-related cardiac, respiratory, neurological complications were extracted from the intraoperative anesthesia monitoring form, postoperative notes, follow-up notes in the ward, anesthesia block forms, and discharge summary. Wound-related complications, including infection, dehiscence, bleeding, and hematoma, were recorded from first postoperative follow-up notes, and data on AVF maturation was recorded from clinic notes on six to eight-week follow-up, dialysis unit charts, and subsequent follow-ups. Any unplanned readmissions, AVF related complications, interventions to salvage fistula, and patency rates recorded until one-year follow-up. In cases of fistula abandonment, the cause of abandonment, including death, transplant, and access failure, and the causes of access failure were also explored. Maturation time was defined as time duration (in weeks) from the creation of the fistula to the day the fistula was palpable with discernible margins and good thrill and used successfully with two needles providing prescribed dialysis for more than two-thirds of dialysis sessions within four consecutive weeks [16] while patency was defined as the time period from the creation of fistula till the time it's blocked or abandoned [16].

Data were analyzed on STATA version 14.2 (StataCorp, College Station, TX). Descriptives of both groups were compared by the independent T-test or Mann Whitney U test for continuous variables and the chi-square or Fisher exact test for categorical variables where appropriate. Kaplan-Meier survival analysis and the logrank test were used to compare patency rates. A p-value of $<0.05$ is considered statistically significant.

\section{Results}

One-hundred fifty-two (152) patients who underwent single-stage BT AVF were included in the analysis. 


\section{Cureus}

One-hundred ten $(110 ; 72.37 \%)$ patients underwent the procedure under GA while $42(27.63 \%)$ patients were operated under RA. Thirty-five (35; 83.33\%) patients had successful RA. Patients' basic demographics and clinical factors are shown in Table 1 . The female gender was more common in the overall study population. Patients in RA were elderly as compared to GA with a mean age of $59.8 \pm 11.2$ vs. $53.3 \pm 14.6(\mathrm{p}=0.010)$. The RA group patients had a higher proportion of diabetes (DM), ischemic heart disease (IHD), and American Society of Anesthesiologists (ASA) class IV compared to the GA group. Other comorbids were comparable between the groups.

\begin{tabular}{|c|c|c|c|}
\hline \multirow{2}{*}{ Variable } & \multicolumn{2}{|l|}{ Anesthesia } & \multirow{2}{*}{ P-value } \\
\hline & General $n=110$ & Regional $n=42$ & \\
\hline Age (years) \pm SD & $53.3 \pm 14.6$ & $59.8 \pm 11.2$ & 0.010 \\
\hline BMI $\left(\mathrm{kg} / \mathrm{m}^{2}\right) \pm \mathrm{SD}$ & $26.6 \pm 6.5$ & $26.9 \pm 4.9$ & 0.802 \\
\hline \multicolumn{4}{|l|}{ Gender } \\
\hline Male n (\%) & $45(40.9)$ & $15(35.7)$ & 0.584 \\
\hline Female n (\%) & $65(59.1)$ & $27(64.3)$ & \\
\hline \multicolumn{4}{|l|}{ Comorbids } \\
\hline -Diabetes n (\%) & $66(60.0)$ & $33(78.6)$ & 0.037 \\
\hline -Duration of Diabetes ( years) & $18.92 \pm 7.82$ & $18.79 \pm 7.82$ & 0.944 \\
\hline -Hypertension n (\%) & $100(90.9)$ & $41(97.6)$ & 0.291 \\
\hline -Ischemic Heart Disease n (\%) & $26(23.6)$ & 25 (59.5) & $<0.001$ \\
\hline -CVA n (\%) & $10(9.1)$ & $4(9.5)$ & 0.934 \\
\hline -Tobacco User n (\%) & $12(10.9)$ & $4(9.5)$ & 0.803 \\
\hline \multicolumn{4}{|l|}{ ASA Class } \\
\hline Class III n (\%) & 104 (94.5) & 33 (78.6) & 0.003 \\
\hline Class IV n (\%) & $6(5.5)$ & $9(21.4)$ & \\
\hline \multicolumn{4}{|l|}{ Primary Renal Pathology } \\
\hline Nephroangiosclerosis & $26(23.6)$ & $9(21.4)$ & 0.187 \\
\hline Adult Polycystic Kidney Disease & $4(3.6)$ & $0(0)$ & \\
\hline Diabetes Mellitus & $59(53.6)$ & $31(73.8)$ & \\
\hline Obstructive Nephropathy or Lithiasis & $5(4.5)$ & $1(2.4)$ & \\
\hline Malformative Pathologies & $1(0.9)$ & $0(0)$ & \\
\hline Unknown Origin & $11(10)$ & $0(0)$ & \\
\hline Dialysis Dependent & 72 (65.45) & $25(59.52)$ & 0.310 \\
\hline Previous Access Surgery & $68(61.8)$ & $22(52.4)$ & 0.290 \\
\hline \multicolumn{4}{|l|}{ Side of Previous Access } \\
\hline Ipsilateral & $35(51.5)$ & $12(54.5)$ & 0.960 \\
\hline Contralateral & $19(27.9)$ & $6(27.3)$ & \\
\hline Multiple Access Surgeries & $14(20.6)$ & 4 (18.2) & \\
\hline Previous Central-Line Cannulation & $73(66.4)$ & $31(73.8)$ & 0.438 \\
\hline \multicolumn{4}{|l|}{ Central cannulation type } \\
\hline -Bilateral / Ipsilateral & $38(52.1)$ & 13 (41.9) & 0.564 \\
\hline -Contralateral & 34 (46.6) & $17(54.8)$ & \\
\hline
\end{tabular}




\section{Cureus}

\begin{tabular}{|c|c|c|c|}
\hline -Femoral & $1(1.4)$ & $1(3.2)$ & \\
\hline Basilic Vein Diameter $(\mathrm{mm}) \pm \mathrm{SD}$ & $3.2 \pm 0.29$ & $3.2 \pm 0.19$ & 0.115 \\
\hline Brachial Artery Diameter $(\mathrm{mm}) \pm \mathrm{SD}$ & $3.5 \pm 0.69$ & $3.7 \pm 0.78$ & 0.782 \\
\hline Operative Time (minutes) & $151 \pm 40.6$ & $140.3 \pm 28$ & 0.122 \\
\hline
\end{tabular}

\section{TABLE 1: Baseline demographics and clinical characteristics of the study population ( $n=152)$}

CVA: cerebrovascular accident; ASA: American Society of Anesthetists

There was no significant difference between groups in terms of dialysis dependency, previous access surgeries, previous history of central and ipsilateral central cannulation, or the size of the basilic vein and brachial artery.

Outcome variables are given in Table 2. Median follow-up duration was comparable in both the groups; 8.3 (IQR) $(7.6,9.1)$ vs. $8.0(7.5,8.8)$ months, $\mathrm{p}=0.852$. There is trend toward higher maturation rates $(97.6 \%$ vs. $92.1 \%, p=0.186)$, patency rates at $6(85.0 \%$ vs. $76.8 \%, p=0.359)$, and one year $(62.5 \%$ vs. $56.6 \%, p=0.327)$ in the RA vs. GA groups (Table 2). Kaplan-Meier curves endorse the same trends of lower failure and higher patency in the RA group as compared to the GA; however, the log-rank p-value was not significant; $p=0.508$ (Figure 1).

\begin{tabular}{|c|c|c|c|}
\hline \multirow{2}{*}{ Variable } & \multicolumn{2}{|l|}{ Anesthesia } & \multirow{2}{*}{ P-value } \\
\hline & General $n=110$ & Regional $n=42$ & \\
\hline \multicolumn{4}{|l|}{ Maturation } \\
\hline Matured & $93(92.1)$ & 40 (97.6) & 0.186 \\
\hline Failed & $8(7.9)$ & $1(2.4)$ & \\
\hline \multicolumn{4}{|l|}{ Patency at 6 months } \\
\hline -Patent & 76 (76.8) & $34(85.0)$ & 0.359 \\
\hline Abandoned & $23(23.2)$ & $6(15.0)$ & \\
\hline \multicolumn{4}{|l|}{ Patency at 1 year } \\
\hline Patent & $56(56.6)$ & $25(62.5)$ & 0.327 \\
\hline Abandoned & $43(43.4)$ & $15(37.5)$ & \\
\hline Cause of abandonment & & & 0.017 \\
\hline Death & $13(30.2)$ & $10(66.7)$ & \\
\hline Access failure & $30(69.8)$ & $5(33.3)$ & \\
\hline Transplant & $0(0)$ & $0(0)$ & \\
\hline Cause of access failure & & & 0.739 \\
\hline Thrombosis & $23(76.7)$ & $4(80)$ & \\
\hline Poor flow & $6(20)$ & $1(20)$ & \\
\hline Aneurysm & $1(3.3)$ & $0(0)$ & \\
\hline AVF complications & $20(20.2)$ & $7(17.5)$ & 0.816 \\
\hline \multicolumn{4}{|l|}{ AVF complications type } \\
\hline Stenosis & $15(75)$ & $7(100)$ & \\
\hline Steal syndrome & 3 (15) & $0(0.0)$ & \\
\hline Bleeding/Hematoma formation & $2(10)$ & $0(0.0)$ & \\
\hline Intervention & $18(18.2)$ & $5(12.8)$ & 0.448 \\
\hline
\end{tabular}




\section{Cureus}

$\begin{array}{llll}\text { Type of Intervention } & & & \\ \text { Revision of anastomosis } & 5(27.8) & 0(0.0) & 0.138 \\ \text { Thrombectomy +/- Revision } & 1(5.6) & 2(40.0) & 3(60.0) \\ \text { Venoplasty } & 10(55.6) & 0(0.0) & 0.443 \\ \text { Others } & 2(11.1) & 4(80) & 0.032 \\ \text { Fistula salvaged after intervention } & 11(61.1) & 3(7.1) & 0.845 \\ \text { Complications of anesthesia* } & 1(0.9) & 1(1-9) & 0.739 \\ \text { Length of stay median days (IQR)‡ } & 1(0-4) & 4(9.5) & 0.075 \\ \text { HDU stay } & 6(5.5) & 2(4.8) & 0.852 \\ \text { ICU stay } & 0(00) & 8.0(7.5,8.8) & \\ \text { Median follow-up time (months) (IQR) } & 8.3(7.6,9.1) & & \end{array}$

TABLE 2: Comparison of BT AVF outcomes among general vs. regional anesthesia, $n=152$

*All were pulmonary complications in both groups.

‡Postoperative length of stay

BT AVF: basilic transposition arteriovenous fistula; HDU: high dependency unit



FIGURE 1: Kaplan Meier's plots showing a comparison of the patency rates of AVF over 12 months in the GA vs. RA groups

AVF: arteriovenous fistula; GA: general anesthesia; RA: regional anesthesia

The rate of abandonment was higher in GA compared to the RA group (43.4\% vs. 37.5\%). The difference between the reasons for abandonment was statistically significant in the two groups. Death was the most common cause of abandonment in the RA group (66.7 vs. $30.2 \%$ ) while access failure was the most common cause of abandonment in the GA group (69.8 vs. 33.3\%). The most common cause of access failure in both groups was thrombosis, followed by poor flow.

Overall complication rates were comparable in both the groups ( 20.2 vs. $17.5 \%, \mathrm{P}=0.816$ ), with common complications being stenosis, steal syndrome, and hematoma formation/bleeding. A trend of a higher rate of interventions was noted in the GA vs. RA group (18.2 vs. $12.8 \%$ ). A higher rate of fistula salvage (80\% vs. 61.1\%) was noted in RA after an intervention. A significantly higher number of patients in RA developed 
anesthesia-related pulmonary complications as compared to GA (7.1 vs. 0.9). No significant difference in total length of hospital stay or high dependency unit (HDU) stay in both groups but a significantly higher rate of ICU stay was noted in RA as compared to the GA group (4.8 vs. $0 \%$ ).

\section{Discussion}

In this study, we compared the outcomes of BT AVF in RA vs. GA in terms of maturation, patency, and complications. RA success rates in our study are comparable to those reported in the literature [17]. A trend of higher maturation rates and higher six-month and 12-month patency rates was observed in patients undergoing procedures in RA. Excellent maturation rates are seen in both the exposure groups $(92.1 \%$ vs. 97.6\% in GA vs. RA, respectively) with very low primary failures, reflecting higher maturation rates than reported by Al-Jaishi AA et al. and Voorzat et al. for upper arm AVFs [6,18]. Al-Jaishi et al. has conducted a meta-analysis on the patency of arteriovenous fistulae, including both forearm and upper arm AVF [6] while Voorzat et al. has investigated patency outcomes as well as complications in both AVFs and arteriovenous grafts (AVGs) [18]. The reason for higher maturation in our study group may be because we have studied only BT AVF as compared to Al-Jaishi et al. and Voorzat et al. [6,18] who have studied BC AVF as well as BT AVF together and BT AVF is reported to have higher maturation rates than BC AVF [19]. One-year patency in our study is comparable to that reported by Al-Jaishi et al. and Voorzat et al. reported estimates [6,18]. Aitken et al. conducted a randomized controlled trial comparing RA vs. local anesthesia (LA) in patients undergoing RC AVF and BC AVFs and concluded improved patency at three months in AVFs performed under RA [20]. Recently, Jorgensen MS et al. have compared the patency of 238 AVFs and AVGs performed under RA vs. GA, and they also concluded decreased failure rates at two months, with RA compared to GA [15]. Both of the above-mentioned studies are limited by short-term follow-up of three and two months, respectively. Our findings are similar to Jorgensen MS et al. and Aitken E et al. [15,20] showing a trend toward higher patency rates in the RA group at six and 12 months. The RA group in our study had a high proportion of elderly, ASA IV, and IHD patients compared to GA, and this proportion is also higher as compared to reported literature $[15,18,21]$. Keeping in view the high proportion of elderly and morbid patients in RA, this trend of higher maturation and patency rates is an important finding and may result in statistically significant differences if both groups were comparable in terms of demographics and clinical characteristics. Jorgensen MS et al. [15] found death as the most common cause of abandonment of AVF in both the RA as well as GA groups, but in our study, death is the most common cause of abandonment in the RA group while access failure is the most common cause of abandonment in the GA group. This finding again indicates that RA was chosen for patients who were morbid having significantly higher comorbids and ASA IV.

Although Robert J et al. [22] found decreased complication rates in RA as compared to GA, in our study, similar to Jorgensen MS et al. [15] and Cole NM et al. [21], we found no difference between the two techniques in terms of AVF-related complication rates. Although none of the patients developed cardiac complications peri-operatively, contrary to our assumption, three (7.1\%) patients in the RA group developed respiratory complications as compared to one $(0.9 \%)$ patient in the GA group in the postoperative period. Two of the patients in the RA group, developing respiratory complications, needed ICU stay while no patient in the GA group needed ICU stay. This higher number of pulmonary complications and ICU stay in the RA group may be explained because of the high number of elderly and morbid patients in the RA group as compared to the younger and less morbid population of the GA group. No difference was observed in the rate of interventions to salvage the fistulae between the two groups but a trend of higher post-intervention salvage rate was seen in the RA group. Cole NM et al. [21] found decreased hospital stay with RA as compared to GA, but in our study, we found no difference in length of stay between the two groups.

This study will fill gaps in loco-regional data on outcomes of BVT AVF when performed under RA vs. GA with some limitations. This is a single-center, retrospective study that carries some inherent limitations, including loss of follow-ups, missing data on potential confounding factors, with the possibility of nonhomogeneous definitions of outcomes recorded by different levels of health professionals over the course of the study. However, this limitation is applied to the whole sampled population.

\section{Conclusions}

Regional anesthesia is a useful technique with potentially improved maturation and patency rates. Nevertheless, the assumed benefit of regional anesthesia in terms of anesthesia-related complications was not observed. A randomized, prospective, multicenter study with a larger number of patients with comparative demographics and clinical risks may overcome the limitations of this study.

\section{Additional Information}

\section{Disclosures}

Human subjects: Consent was obtained or waived by all participants in this study. Ethics Review Committee, The Aga Khan University issued approval 2020-5601-14962. Ziaur Rehman: Outcomes of single stage basilic transposition arteriovenous fistula under regional anesthesia versus general anesthesia: A single center, retrospective cohort study. Thank you for your application for exemption from ethical approval regarding the above mentioned study. Your study was reviewed and approved as exemption. Please ensure that the study is performed as per protocol following all AKU standards. You may proceed with the 
study. Animal subjects: All authors have confirmed that this study did not involve animal subjects or tissue. Conflicts of interest: In compliance with the ICMJE uniform disclosure form, all authors declare the following: Payment/services info: All authors have declared that no financial support was received from any organization for the submitted work. Financial relationships: All authors have declared that they have no financial relationships at present or within the previous three years with any organizations that might have an interest in the submitted work. Other relationships: All authors have declared that there are no other relationships or activities that could appear to have influenced the submitted work.

\section{References}

1. Imai E, Matsuo S: Chronic kidney disease in Asia . Lancet. 2008, 371:2147-8. 10.1016/S0140-6736(08)609289

2. Levey AS, de Jong PE, Coresh J, et al.: The definition, classification, and prognosis of chronic kidney disease: a KDIGO Controversies Conference report. Kidney Int. 2011, 80:17-28. 10.1038/ki.2010.483

3. Hasan M, Sutradhar I, Gupta RD, Sarker M: Prevalence of chronic kidney disease in South Asia: a systematic review. BMC Nephrol. 2018, 19:291. 10.1186/s12882-018-1072-5

4. Lacson E Jr, Wang W, Lazarus JM, Hakim RM: Change in vascular access and hospitalization risk in longterm hemodialysis patients. Clin J Am Soc Nephrol. 2010, 5:1996-2003. 10.2215/CJN.08961209

5. Murad MH, Elamin MB, Sidawy AN, et al.: Autogenous versus prosthetic vascular access for hemodialysis: a systematic review and meta-analysis. J Vasc Surg. 2008, 48:34S-47S. 10.1016/j.jvs.2008.08.044

6. Al-Jaishi AA, Oliver MJ, Thomas SM, et al.: Patency rates of the arteriovenous fistula for hemodialysis: a systematic review and meta-analysis. Am J Kidney Dis. 2014, 63:464-78. 10.1053/j.ajkd.2013.08.023

7. Hoggard J, Saad T, Schon D, et al.: Guidelines for venous access in patients with chronic kidney disease. A position statement from the American Society of Diagnostic and Interventional Nephrology, Clinical Practice Committee and the Association for Vascular Access. Semin Dial. 2008, 21:186-91.

8. Dagher F, Gelber R, Ramos E, Sadler J: The use of basilic vein and brachial artery as an A-V fistula for long term hemodialysis. J Surg Res. 197620, 20:373-6. 10.1016/0022-4804(76)90029-9

9. Sheta M, Hakmei J, London M, Wooster M, Aruny J, Ross J, Illig KA: One- versus two-stage transposed brachiobasilic arteriovenous fistulae: a review of the current state of the art. J Vasc Access. 2020, 21:281-6. 10.1177/1129729819862694

10. Lee TH, Marcantonio ER, Mangione CM, et al.: Derivation and prospective validation of a simple index for prediction of cardiac risk of major noncardiac surgery. Circulation. 1999, 100:1043-9.

10.1161/01.cir.100.10.1043

11. Chue KM, Thant KZ, Luo HD, Soh YH, Ho P: Comprehensive comparison of the performance of autogenous brachial-basilic transposition arteriovenous fistula and prosthetic forearm loop arteriovenous graft in a multiethnic Asian hemodialysis population. Biomed Res Int. 2016, 2016:8693278. 10.1155/2016/8693278

12. Shemesh D, Olsha O, Orkin D, Raveh D, Goldin I, Reichenstein Y, Zigelman C: Sympathectomy-like effects of brachial plexus block in arteriovenous access surgery. Ultrasound Med Biol. 2006, 32:817-22. 10.1016/j.ultrasmedbio.2006.02.1420

13. Hingorani AP, Ascher E, Gupta P, et al.: Regional anesthesia: preferred technique for venodilatation in the creation of upper extremity arteriovenous fistulae. Vascular. 2006, 14:23-6. 10.2310/6670.2006.00006

14. Weissman C: The metabolic response to stress: an overview and update . Anesthesiology. 1990, 73:308-27. 10.1097/00000542-199008000-00020

15. Jorgensen MS, Farres H, James BL, et al.: The role of regional versus general anesthesia on arteriovenous fistula and graft outcomes: a single-institution experience and literature review. Ann Vasc Surg. 2020, 62:287-94. 10.1016/j.avsg.2019.05.016

16. Lok CE, Huber TS, Lee T, et al.: KDOQI clinical practice guideline for vascular access: 2019 update . Am J Kidney Dis. 2020, 75:S1-S164. 10.1053/j.ajkd.2019.12.001

17. Williams SR, Chouinard P, Arcand G, Harris P, Ruel M, Boudreault D, Girard F: Ultrasound guidance speeds execution and improves the quality of supraclavicular block. Anesth Analg. 2003, 97:1518-23. 10.1213/01.ane.0000086730.09173.ca

18. Voorzaat BM, Janmaat CJ, van der Bogt KEA, Dekker FW, Rotmans JI: Patency outcomes of arteriovenous fistulas and grafts for hemodialysis access: a trade-off between nonmaturation and long-term complications. Kidney360. 2020, 1:916-24. 10.34067/KID.0000462020

19. Hakaim AG, Nalbandian M, Scott T: Superior maturation and patency of primary brachiocephalic and transposed basilic vein arteriovenous fistulae in patients with diabetes. J Vasc Surg. 1998, 27:154-7. 10.1016/s0741-5214(98)70302-7

20. Aitken E, Jackson A, Kearns R, Steven M, Kinsella J, Clancy M, Macfarlane A: Effect of regional versus local anaesthesia on outcome after arteriovenous fistula creation: a randomized controlled trial. Lancet. 201610, 388:1067-74. 10.1016/S0140-6736(16)30948-5

21. Cole NM, Vlassakov K, Brovman EY, Heydarpour M, Urman RD: Regional anesthesia for arteriovenous fistula surgery may reduce hospital length of stay and reoperation rates. Vasc Endovascular Surg. 2018, 52:418-26. 10.1177/1538574418772451

22. Beaulieu RJ, Locham S, Nejim B, Dakour-Aridi H, Woo K, Malas MB: General anesthesia is associated with reduced early failure among patients undergoing hemodialysis access. J Vasc Surg. 2019, 69:890-897.e5. 10.1016/j.jvs.2018.05.247 